\title{
Clinical characteristics and neuroimaging findings in eagle syndrome induced internal jugular vein stenosis
}

\author{
Chaobo Bai ${ }^{1,2,3}$, Zhongao Wang ${ }^{1,2,3}$, Jingwei Guan ${ }^{1,2,3}$, Kexin Jin ${ }^{1,2,3}$, Yuchuan Ding ${ }^{3,4}$, Xunming Ji ${ }^{2,3,5}$, \\ Ran Meng ${ }^{1,2,3}$
}

${ }^{1}$ Department of Neurology, Xuanwu Hospital, Capital Medical University, Beijing 100000, China; ${ }^{2}$ Advanced Center of Stroke, Beijing Institute for Brain Disorders, Beijing 100000, China; ${ }^{3}$ Department of China-America Institute of Neuroscience, Xuanwu Hospital, Capital Medical University, Beijing 100000, China; ${ }^{4}$ Department of Neurosurgery, Wayne State University School of Medicine, Detroit, MI, USA; ${ }^{5}$ Department of Neurosurgery, Xuanwu Hospital, Capital Medical University, Beijing 100000, China

Contributions: (I) Conception and design: C Bai, R Meng; (II) Administrative support: X Ji, Y Ding, R Meng; (III) Provision of study materials or patients: C Bai, Z Wang, J Guan, K Jin; (IV) Collection and assembly of data: C Bai, Z Wang, J Guan, K Jin; (V) Data analysis and interpretation: C Bai, R Meng; (VI) Manuscript writing: All authors; (VII) Final approval of manuscript: All authors.

Correspondence to: Ran Meng. Xuanwu Hospital, Capital Medical University, Beijing 100000, China. Email: victor65@126.com.

\begin{abstract}
Background: Eagle syndrome is a condition that causes pharyngeal pain, facial pain, swallowing difficulties, and symptoms of arterial impingement due to the elongated styloid process. However, few reports were about eagle syndrome with venous compression up to now. This study aimed to identify the clinical profiles of the internal jugular vein stenosis (IJVS) related eagle syndrome comprehensively.

Methods: A total of 27 patients, who were diagnosed as IJVS induced by styloid process compression were enrolled. The clinical manifestations and imaging features were analyzed.

Results: Styloid process compression was presented in all of the 27 IJVS patients, in which, the top three symptoms included insomnia (81.5\%), tinnitus $(63.0 \%)$ and head noises $(63.0 \%)$. The most vulnerable segment of internal jugular vein (IJV) was J3 segment (96.3\%). The average styloid process length in our study was $3.7 \mathrm{~cm}$. Hearing impairment was more common in bilateral IJVS (68.8\% vs. 18.2\%, P=0.018). One patient reported significant relief of symptoms at 1 year follow-up after underwent styloidectomy combined with stenting.

Conclusions: Neurological symptoms of eagle syndrome induced IJVS were various, including either arterial or venous issues. Better understanding of this disease entity may be helpful for clinical diagnosis and treatment.
\end{abstract}

Keywords: Internal jugular vein (IJV); eagle syndrome; osseous compression; styloid process compression; internal jugular vein stenosis (IJVS)

Submitted Oct 22, 2019. Accepted for publication Dec 03, 2019.

doi: $10.21037 /$ atm.2019.12.93

View this article at: http://dx.doi.org/10.21037/atm.2019.12.93

\section{Introduction}

Eagle syndrome (also known as styloid syndrome), was described by American otolaryngologist Watt Weems Eagle in 1937 for the first time, which is also a rare condition that usually characterizes a sudden, sharp nerve pain, such as the pharynx, the back of the throat, and the base of the tongue, triggered by swallowing, moving the jaw, or turning the neck. This entity was usually mediated by elongated styloid processes (the slender pointed skull below the ear), and/ or calcification of the stylohyoid ligament, which interferes with the function of the peripheral nerves, causing pain and difficulty in swallowing (1-3). The $3 \mathrm{D}$ reconstruction of CT scanning is recommended to visualize elongated 
styloid processes $(4,5)$. Surgical removal of elongated styloid processes is currently the most common treatment for eagle syndrome (6).

In addition, elongated styloid processes can also impinge the surrounding blood vessels. The vascular eagle syndrome is usually caused by elongated styloid process that impinges the internal carotid artery. In this condition, the blood flow of the internal carotid artery is restricted by external impingement, resulting in transient ischemic attack and stroke $(7,8)$. However, few reports were about the eagle syndrome with venous compression. In this study, we aimed to identify the clinical profiles of the internal jugular vein stenosis (IJVS) related eagle syndrome comprehensively in an attempt to gain better understanding of this syndrome and provide reference for clinical diagnosis and treatment.

\section{Methods}

This study was conducted and approved by the Institutional Ethic Committee of the Xuanwu Hospital, Capital Medical University, Beijing, China. All patients signed informed consent before enrolling. From October 2017 to October 2018, a total of 27 patients, were diagnosed as IJVS induced by elongated styloid process were enrolled in this single center real-world study.

IJVS patients with eagle syndrome should meet the following criteria: (I) The stenotic IJV segment of the unilateral or bilateral IJV was more than $50 \%$ compared to the proximal adjacent IJV segment, which can be visualized at contrast-enhanced magnetic resonance venography (CE-MRV), computed tomographic venography (CTV) or catheter venography (CV). (II) Abnormally distorted collateral vessels were confirmed by CE-MRV, CTV or CV. (III) Patients presented with nonspecific clinical symptoms: headache, tinnitus, head noises, visual disorders, hearing loss, memory loss, insomnia, anxiety and neck discomfort. (IV) IJVS secondary to elongated styloid process compression was confirmed by CTV and $3 \mathrm{D}$ reconstruction of CT scanning.

The exclusion criteria were the following: (I) No abnormally distorted collateral vessels were found in CEMRV, CTV or CV. (II) The measured length of styloid process at $3 \mathrm{D}$ reconstruction of CT scanning was less than $3 \mathrm{~cm}$, which was inconsistent with the diagnostic criteria of Eagle syndrome. (III) The symptoms could be explained by other diseases, such as pre-existing ophthalmology or otology diseases (IV) CNS malignancy. (V) Patients with incomplete clinical data.
All patients received standard medical treatment after admission, including antiplatelet and standard anticoagulant therapy, dehydration, and other symptomatic treatments to alleviate clinical symptoms. Given the clinical consensus and guidelines for surgical intervention of IJVS associated eagle syndrome is lacking, elongated styloid processes resection and subsequent balloon dilation and/or stenting were performed only when the patient strongly requested surgery.

Social Science Statistical Software Package (SPSS) version 21.0 program was applied for this study. Continuous variables that conform to Gaussian distribution are represented by mean \pm standard deviation. Otherwise, they are represented as median (interquartile range). Comparison of continuous variables was calculated by $t$ test or by MannWhitney $\mathrm{U}$ test. Categorical variables were depicted as counts and percentages, which were analyzed by chi-square test. Two-sided $\mathrm{P}$ values $<0.05$ were considered a statistically significant difference.

\section{Results}

From October 2017 to October 2018 in Neurology Department, Xuanwu Hospital (Beijing, China), a total of 133 consecutive patients who met the IJVS diagnostic criteria were enrolled. In which, 27 IJVS cases matched the diagnostic criteria for Eagle Syndrome were into the final analysis, included 15 males and 12 females. The mean age was $59.7 \pm 11.3$ years. The onset-to-door time was $3(1-10)$ years.

In this cohort, the top three symptoms were insomnia $(22 / 27,81.5 \%)$, tinnitus $(17 / 27,63.0 \%)$ and head noises $(17 / 27,63.0 \%)$, respectively. Other clinical symptoms included hearing impairment $(13 / 27,48.1 \%)$, visual disorder $(8 / 27,29.6 \%)$, headache $(12 / 27,44.4 \%)$, neck discomfort $(7 / 27,26.9 \%)$, memory decline $(3 / 27,11.1 \%)$ and anxiety $(3 / 27,11.1 \%)$. With regard to comorbid diseases, hyperlipidemia $(7 / 27,26.9 \%)$ and hypertension $(6 / 27$, $22.2 \%)$ were the top two issues, followed by diabetes $(1 / 27$, $3.7 \%)$, hyperhomocysteinemia $(1 / 27,3.7 \%)$, Hyperuricemia $(2 / 27,7.4 \%)$, cerebral venous sinus thrombosis (CVST) $(3 / 27,11.1 \%)$. In addition, alcohol abuse was found in 4 $(14.8 \%)$ patients, and smoking was in $3(11.1 \%)$ patients. During our follow-up, patients responded poorly to standard medical treatment. Only 6 patients reported improvement in clinical symptoms. However, 11 reported no change, and 10 reported exacerbations. Details were shown in Table 1 . 
Table 1 Baseline clinical data

\begin{tabular}{|c|c|}
\hline Clinical features & N (\%) \\
\hline \multicolumn{2}{|l|}{ Demographics } \\
\hline No. of patients & 27 \\
\hline Gender (male/female) & $15 / 12$ \\
\hline Age (y) & $59.7 \pm 11.3$ \\
\hline Onset-to-door time (y) & $3[1-10]$ \\
\hline \multicolumn{2}{|l|}{ Comorbid diseases } \\
\hline Hypertension & $6(22.2)$ \\
\hline Diabetes & $1(3.7)$ \\
\hline Hyperlipidemia & $7(26.9)$ \\
\hline Hyperhomocysteinemia & $1(3.7)$ \\
\hline Hyperuricemia & $2(7.4)$ \\
\hline CVST & $3(11.1)$ \\
\hline \multicolumn{2}{|l|}{ Life habit } \\
\hline Alcohol abuse & $4(14.8)$ \\
\hline Smoking & $3(11.1)$ \\
\hline \multicolumn{2}{|l|}{ Clinical manifestations } \\
\hline Insomnia & $22(81.5)$ \\
\hline Hearing impairment & $13(48.1)$ \\
\hline Visual impairment & $8(29.6)$ \\
\hline Headache & $12(44.4)$ \\
\hline Tinnitus & $17(63.0)$ \\
\hline Head noises & $17(63.0)$ \\
\hline Neck discomfort & $7(26.9)$ \\
\hline Memory decline & $3(11.1)$ \\
\hline Anxiety & $3(11.1)$ \\
\hline \multicolumn{2}{|l|}{ Follow up } \\
\hline Improve & $6(22.2)$ \\
\hline No change & $11(40.7)$ \\
\hline Worse & $10(37.1)$ \\
\hline
\end{tabular}

CVST, cerebral venous sinus thrombosis.

In our study, we observed that the $\mathrm{J} 3$ segment is the most vulnerable segment of IJVS $(26 / 27,96.3 \%)$, followed by J2 segment (4/27, 14.8\%) and J1 segment (2/27, 7.4\%). In which, $74.1 \%(20 / 27)$ of patients were due to transverse mass of $\mathrm{C} 1$ combined with styloid process compression. $25.9 \%(5 / 27)$ is due to styloid process compression.
Table 2 Characteristics of IJVS on MRV, 3D-computer tomography imaging

\begin{tabular}{lc}
\hline Imaging characteristics & $\mathrm{N}(\%)$ \\
\hline Stenotic segments & $2(7.4)$ \\
$\mathrm{J} 1$ & $4(14.8)$ \\
$\mathrm{J} 2$ & $26(96.3)$ \\
$\mathrm{J} 3$ & \\
Types of compression (n, \%) & $20(74.1)$ \\
Transverse mass of C1 combined with & \\
styloid process compression & $7(25.9)$ \\
Styloid process compression & \\
Stenotic sites (n, \%) & $21(77.8)$ \\
Left & $22(81.5)$ \\
Right & $16(59.3)$ \\
Bilateral & 3.7 \\
Average styloid process length (cm)
\end{tabular}

Table 3 Clinical manifestations between different segments of IJVS

\begin{tabular}{lllc}
\hline Clinical manifestations & \multicolumn{1}{c}{$\mathrm{J} 1$} & $\mathrm{~J} 2$ & $\mathrm{~J} 3$ \\
\hline Insomnia & $2(9.1)$ & $4(18.2)$ & $21(95.5)$ \\
Hearing impairment & $1(7.7)$ & $1(7.7)$ & $13(100.0)$ \\
Visual impairment & $1(12.5)$ & $1(12.5)$ & $8(100.0)$ \\
Headache & $0(0.0)$ & $0(0.0)$ & $12(100.0)$ \\
Tinnitus & $2(11.8)$ & $3(17.6)$ & $17(100.0)$ \\
Head noises & $0(0.0)$ & $2(11.8)$ & $16(94.1)$ \\
Neck discomfort & $1(14.3)$ & $2(28.6)$ & $6(85.7)$ \\
Memory decline & $1(33.3)$ & $1(33.3)$ & $3(100.0)$ \\
Anxiety & $0(0.0)$ & $0(0.0)$ & $3(100.0)$ \\
\hline
\end{tabular}

Additionally, bilateral IJVS stenosis occurred in 16 patients (16/27, 59.3\%). In addition, the left stenosis was found in 21 patients $(21 / 27,77.8 \%)$ and the right stenosis was found in 22 patients $(22 / 27,81.5 \%)$. Average styloid process length which was measured at $3 \mathrm{D}$ reconstruction of CT scanning was $3.7 \mathrm{~cm}$. Subgroup analysis of clinical manifestation between different stenotic segments was depicted, in which the J3 segment was closely related to various clinical manifestations. Details were depicted in Tables 2,3.

The comparisons of demographic and clinical manifestations between unilateral and bilateral IJVS were 
Table 4 Comparisons between unilateral and bilateral IJV stenosis

\begin{tabular}{|c|c|c|c|}
\hline & Unilateral stenosis & Bilateral stenosis & $P$ value \\
\hline No. of patients & 11 & 16 & NA \\
\hline Age (y) & $61.4 \pm 8.6$ & $58.6 \pm 13.1$ & 0.549 \\
\hline Onset-to-door time (y) & $4[1-19]$ & $2[2-6]$ & 0.414 \\
\hline \multicolumn{4}{|l|}{ Clinical manifestation } \\
\hline Insomnia & $10(90.9)$ & $12(75.0)$ & 0.618 \\
\hline Hearing impairment & $2(18.2)$ & $11(68.8)$ & $0.018^{*}$ \\
\hline Visual impairment & $3(27.3)$ & 5 (31.3) & 1.000 \\
\hline Head noises & $8(72.7)$ & $9(56.3)$ & 0.448 \\
\hline Neck discomfort & $3(27.3)$ & $4(25.0)$ & 1.000 \\
\hline Memory decline & $2(18.2)$ & $1(6.3)$ & 0.549 \\
\hline Anxiety & $1(9.1)$ & $2(12.5)$ & 1.000 \\
\hline
\end{tabular}

NA, not applicable; IJV, internal jugular vein. *, Statistically significant at $\mathrm{P}<0.05$.

shown in Table 4. The age of symptoms onset, the duration from onset to admission and gender showed no statistic difference between the bilateral and the unilateral IJVS subgroups. Regarding clinical manifestations, hearing impairment was more common in bilateral stenosis compared with unilateral stenosis $(68.8 \%$ vs. $18.2 \%$, $\mathrm{P}=0.018)$. However, other clinical manifestations mentioned above were similar in the two subgroups.

Among the 27 cases, only one patient underwent surgical intervention. This 49-year-old male, who complained of intermittent headaches, persistent head noises and tinnitus for 3 years, was confirmed the diagnosed as severe bilateral IJVS surrounded by abnormally distorted vertebral venous plexuses by CE-MRV in Xuanwu Hospital in November 2017. 3D reconstruction CT maps showed the bilateral IJVS at J3 segments were obviously compressed by the adjacent lateral mass of $\mathrm{C} 1$ and styloid process. The styloid processes length was $4.4 \mathrm{~cm}$ in left and $4.1 \mathrm{~cm}$ in right (Figure 1). The patient underwent bone resection and balloon dilation in left IJV after finished his comprehensively preoperative assessment, as the outflow disorder in left IJV was more severe than that in right. Both the styloid process and the lateral mass of $\mathrm{C} 1$ on the left side were resected and the left stenotic IJV was corrected by balloon dilation. The symptoms were relieved immediately after surgery. More importantly, at one-year follow-up postoperation, all symptoms were disappeared and the previous left stenotic IJV had restored normal blood flow and the abnormally tortuous vertebral venous plexus was also decreased in his follow-up CTV maps (Figure 2).

\section{Discussion}

Eagle syndrome is a rare condition result in pharyngeal pain, facial paresthesia and pain, difficulty swallowing, and symptoms of arterial impingement due to elongation of the styloid process. Several case reports and clinical reviews have reported the clinical and imaging features of typical eagle syndrome (1-6). However, few reports were about eagle syndrome focuses on venous symptoms up to now. In our clinical practice, we have noticed that a few numbers of IJVS showed bony compression, especially elongated styloid processes combined with transverse mass of C1. In an attempt to gain better understanding of this entity and provide reference for clinical diagnosis and treatment, we aimed to depict the clinical manifestations and imaging features of IJVS related eagle syndrome.

In clinical practice, clinical presentation and image 

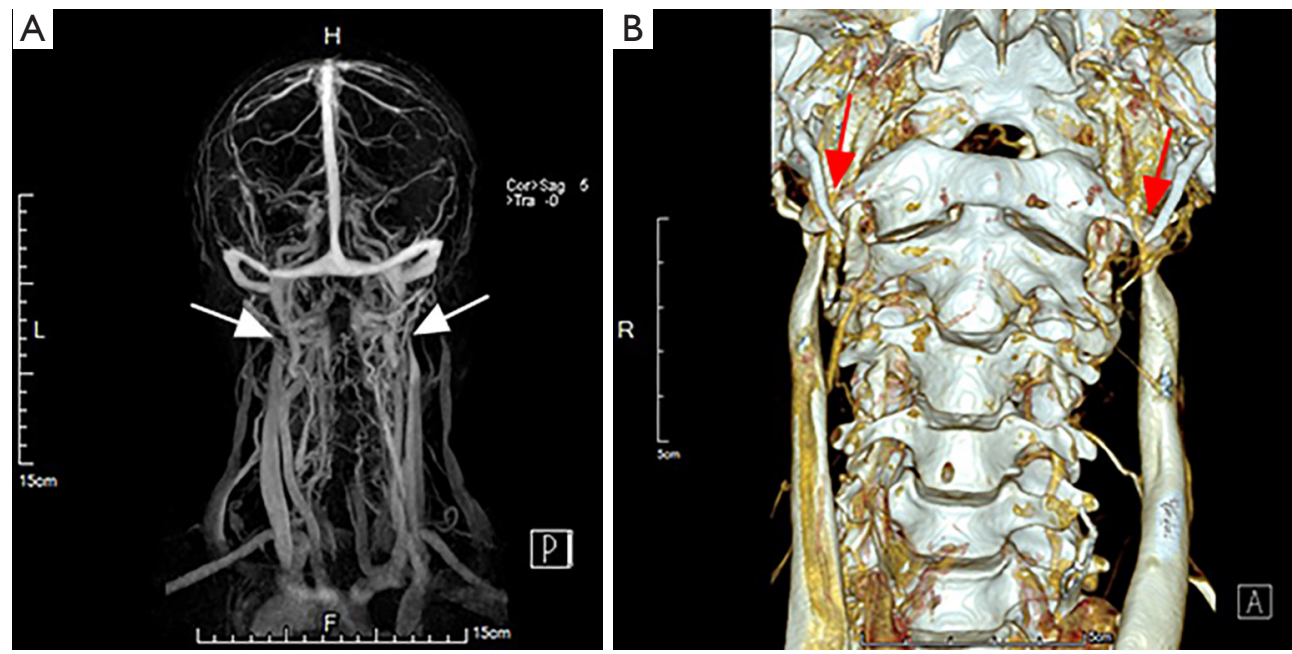

Figure 1 The initial CE-MRV showed severe bilateral IJVS, which was surrounded by abnormally distorted vertebral venous plexuses (A, white arrow). In 3D reconstruction of CT scanning, the J3 segments of the bilateral IJV were obviously compressed by the adjacent lateral mass of C1 and styloid process (B, red arrow). CE-MRV, contrast-enhanced magnetic resonance venography; IJVS, internal jugular vein stenosis.
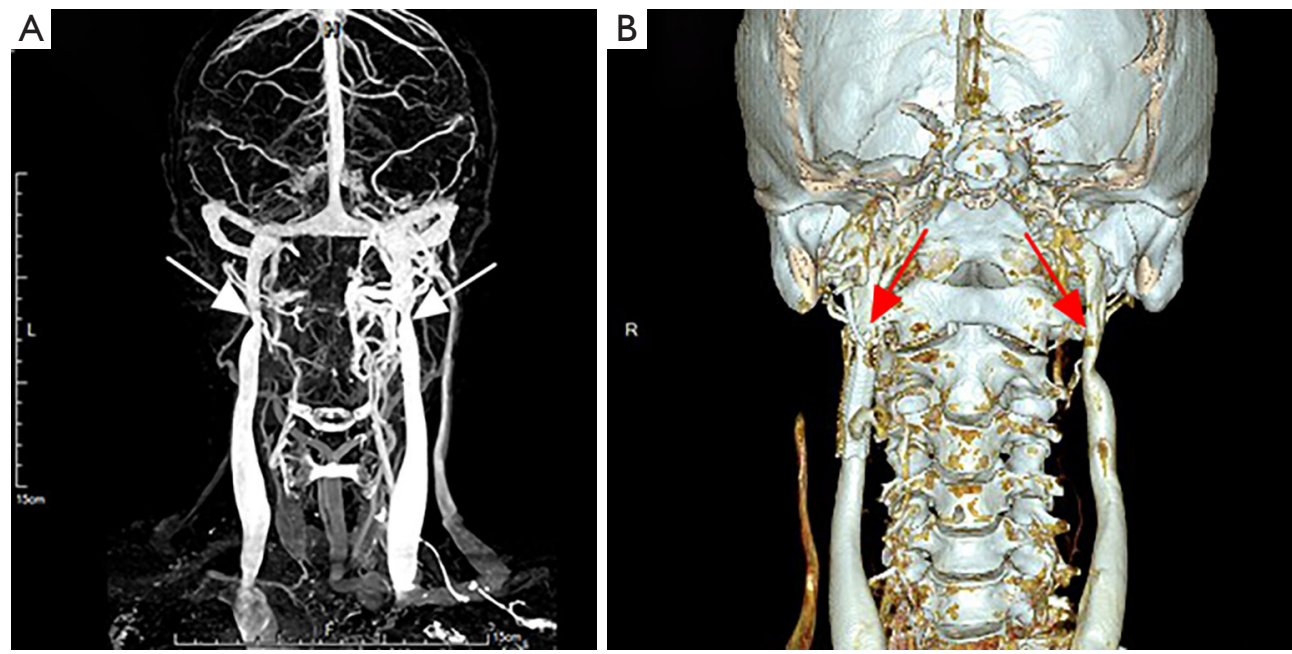

Figure 2 In 1-year follow-up CTV (A, white arrow) and 3D reconstruction of CT scanning (B, red arrow), it could be observed that the previous left stenotic IJV had restored normal blood flow and the abnormally tortuous vertebral venous plexus was also decreased. CTV, computed tomographic venography; IJV, internal jugular vein.

characteristics are essential for the diagnosis of IJVS. Notably, the stenotic IJV and surrounding abnormally tortuous vertebral venous plexus compensation are vital clues for the diagnosis of symptomatic IJVS. However, the unfamiliarity with IJVS and the lack of awareness of this disease entity have resulted in patients being misdiagnosed and delayed treatment in their first-time clinical visiting. We noted that the average age of IJVS patients in our study was in their middle age $(59.7 \pm 11.3$ years $)$, and the time of first diagnosis was several years after symptoms onset (the median time from symptoms onset to confirm the diagnosis was 3 years). In addition to depicting the clinical profiles of eagle syndrome related IJVS for the first time, this study also provides a reference to clinicians who are not familiar with this entity, to avoid misdiagnosis and treatment delay.

Many literatures have reported that various CNS diseases such as multiple sclerosis, Alzheimer's disease, and Parkinson's disease may be associated with IJVS (9-12). The 
underlying pathological mechanism of IJVS is unclear but has aroused great interests among researchers. Notably, clinical symptoms in our study such as insomnia, tinnitus, head noises, hearing impairment, visual impairment, headache, neck discomfort, memory decline and anxiety were similar to the previously reported non-external compression IJVS cohort description $(13,14)$. This may suggest that IJVS with different etiologies may share the same pathological mechanism regardless of the presence of external compression.

With the development of imaging technology, it is not difficult to discover stenotic IJV induced by osseous compression below the skull base in the routine examination. Previous studies have reported that the proportion of osseous compression in Chinese IJVS accounts for about $40 \%(13-15)$. In which, the transverse process of $\mathrm{C} 1$ and the styloid process are the most common factors of bony compression. Differing from previous studies, our study revealed that elongated styloid process compressed not only artery but also venous wall, resulting in neurological disorder. To our knowledge, this is the report for the first time. In addition, IJV is more susceptible to be compressed by adjacent structures due to lack of smooth muscle, especially abnormal bone structures, such as the transverse process of $\mathrm{C} 1$ and the styloid process.

IJV can be divided into three segments, the upper (J3), the middle (J2) and the inferior segment (J1). In which, the $\mathrm{J} 3$ segment is the most vulnerable segment. Our study found that $96.3 \%$ of IJVS occurred in J3 segment, which is consistent with previous IJVS cohort studies (13-15). These findings suggest that $\mathrm{J} 3$ segment is a vital part of the connection to cerebral venous sinus, as a result, its blood flow shear stress is larger than the other two segments, so the vascular endothelium is more susceptible to damage. More importantly, the $\mathrm{J} 3$ segment of IJV is close to many bony structures, such as the transition process of $\mathrm{C} 1$ and the styloid process, thus is more susceptible to be compressed by adjacent abnormal bony structures.

Our study revealed that the percentage of patients with unilateral stenosis was similar to bilateral stenosis. In addition, the age, symptoms onset to door time, and gender distribution were similar between the two patients' subgroups. With regard to clinical symptoms, hearing impairment was more common in bilateral stenosis subgroup. This may suggest that bilateral IJVS may cause more severe blood flow circulatory disturbances in auditoryrelated structures.
Previous literature on stenting has been controversial for the efficacy of IJVS Caucasians with multiple sclerosis (16-21). In our previous cohort study, we found that nonexternal-compressed IJVS Chinese in the absence of multiple sclerosis was significantly able to benefit from stenting (22). However, it is worth noting that for an identified external compression IJVS, simple stenting may be ineffective or even harmful. For extrinsic impingement of the IJVS, simple stenting may present an adverse event in which the balloon cannot well expand and block the IJV blood flow (23-25). In this condition, the decompressive bone resection may become the first choice for bony compression induced IJVS. In addition, in a recent work, the author underlines the importance of "dynamic imaging in suspected Eagle Syndrome" rather than static one on eagle syndrome related IJVS diagnosis $(26,27)$. In our study, only one patient received the decompressive bone resection. Although the patient reported symptomatic relief at 1 year of follow-up, our study was unable to draw conclusions about the safety and efficacy of the decompressive bone resection due to the small number of patients included. Accordingly, larger prospective studies are needed to explore the safety and efficacy of decompressive bone resections.

Our study has several limitations. Firstly, the number of patients we included in this study was small, which may only reflect real-world information in our center. Secondly, we did not perform a quantitative analysis of the patient's symptoms, so the comparison of unilateral stenosis with bilateral stenosis may not be accurate enough. Finally, only one patient received decompressive bone resection. A greater prospective trial regarding the safety and efficacy of the decompressive bone resection is urgently needed.

\section{Conclusions}

Neurological symptoms of Eagle syndrome induced IJVS were various. Mainly included insomnia, head noises and tinnitus. The most vulnerable section was J3 segment of IJV. Hearing disorder was more common in patients with bilateral IJVS. A better understanding of this disease entity may provide a great reference for clinicians and avoid misdiagnosis. Decompressive bone resection may be a promising method to treat IJVS related eagle syndrome. However, more prospective trials on the aspects of safety and efficacy of the decompressive bone resection are urgently needed. 


\section{Acknowledgments}

We would like to thank all patients and doctors who participated in this study for their cooperation.

Funding: This study was sponsored by the National Key R\&D Program of China (2017YFC1308401), the National Natural Science Foundation (81371289), the Project of Beijing Municipal Top Talent for Healthy Work of China (2014-2-015).

\section{Footnote}

Conflicts of Interest: The authors have no conflicts of interest to declare.

Ethical Statement: The authors are accountable for all aspects of the work in ensuring that questions related to the accuracy or integrity of any part of the work are appropriately investigated and resolved. This study was conducted and approved by the Institutional Ethic Committee of the Xuanwu Hospital, Capital Medical University, Beijing, China. All patients signed informed consent before enrolling.

\section{References}

1. Kazmierski R, Wierzbicka M, Kotecka-Sowinska E, et al. Expansion of the Classification System for Eagle Syndrome. Ann Intern Med 2018;168:746-7.

2. Bakshi SS. An Unusually Long Process: Eagle Syndrome. Am J Med 2016;129:1166-7.

3. Morrison RJ, Morrison PJ. Aetiology of Eagle syndrome: ossification of the stylohyoid ligament. QJM 2019;112:467.

4. Shah K, Miller DJ. Three-dimensional modeling of Eagle syndrome. Neurology 2016;87:2279-80.

5. Nakagawa D, Ota T, Iijima A, et al. Diagnosis of Eagle syndrome with 3-dimensional angiography and nearinfrared spectroscopy: case report. Neurosurgery 2011;68:E847-9.

6. Shin JH, Herrera SR, Eboli P, et al. Entrapment of the glossopharyngeal nerve in patients with Eagle syndrome: surgical technique and outcomes in a series of 5 patients. J Neurosurg 2009;111:1226-30.

7. Li Z, Hua Y, Yang J, et al. Ultrasound Evaluation of Transient Ischemic Attack Caused by Styloid Process Elongation: A Case Report. Front Neurol 2019;10:26.

8. Chuang WC, Short JH, McKinney AM, et al. Reversible left hemispheric ischemia secondary to carotid compression in Eagle syndrome: surgical and CT angiographic correlation. AJNR Am J Neuroradiol 2007;28:143-5.

9. Zamboni P, Galeotti R, Menegatti E, et al. Chronic cerebrospinal venous insufficiency in patients with multiple sclerosis. J Neurol Neurosurg Psychiatry 2009;80:392-9.

10. Chung CP, Beggs C, Wang PN, et al. Jugular venous reflux and white matter abnormalities in Alzheimer's disease: a pilot study. J Alzheimers Dis 2014;39:601-9.

11. Liu M, Xu H, Wang Y, et al. Patterns of chronic venous insufficiency in the dural sinuses and extracranial draining veins and their relationship with white matter hyperintensities for patients with Parkinson's disease. J Vasc Surg 2015;61:1511-20.e1.

12. Bruno A, Napolitano M, Califano L, et al. The Prevalence of Chronic Cerebrospinal Venous Insufficiency in Meniere Disease: 24-Month Follow-up after Angioplasty. J Vasc Interv Radiol 2017;28:388-91.

13. Zhou D, Ding J, Asmaro K, et al. Clinical Characteristics and Neuroimaging Findings in Internal Jugular Venous Outflow Disturbance. Thromb Haemost 2019;119:308-18.

14. Bai C, Xu Y, Zhou D, et al. The comparative analysis of non-thrombotic internal jugular vein stenosis and cerebral venous sinus stenosis. J Thromb Thrombolysis 2019;48:61-7.

15. Ding JY, Zhou D, Pan LQ, et al. Cervical spondylotic internal jugular venous compression syndrome. CNS Neurosci Ther 2020;26:47-54.

16. Ghezzi A, Annovazzi P, Cocco E, et al. Endovascular treatment of CCSVI in patients with multiple sclerosis: clinical outcome of 462 cases. Neurol Sci 2013;34:1633-7.

17. Ghezzi A, Annovazzi P, Amato MP, et al. Adverse events after endovascular treatment of chronic cerebro-spinal venous insufficiency (CCSVI) in patients with multiple sclerosis. Mult Scler 2013;19:961-3.

18. Zamboni P, Tesio L, Galimberti S, et al. Efficacy and Safety of Extracranial Vein Angioplasty in Multiple Sclerosis: A Randomized Clinical Trial. JAMA Neurol 2018;75:35-43.

19. Siddiqui AH, Zivadinov R, Benedict RH, et al. Prospective randomized trial of venous angioplasty in MS (PREMiSe). Neurology 2014;83:441-9.

20. Jagannath VA, Pucci E, Asokan GV, et al. Percutaneous transluminal angioplasty for treatment of chronic cerebrospinal venous insufficiency (CCSVI) in people with multiple sclerosis. Cochrane Database Syst Rev 2019;5:CD009903.

21. Zivadinov R, Weinstock-Guttman B. Multiple sclerosis: Extracranial venous angioplasty is ineffective to treat MS. 
Nat Rev Neurol 2018;14:129-30.

22. Zhou D, Meng R, Zhang X, et al. Intracranial hypertension induced by internal jugular vein stenosis can be resolved by stenting. Eur J Neurol 2018;25:365-e13.

23. Higgins JN, Garnett MR, Pickard JD, et al. An Evaluation of Styloidectomy as an Adjunct or Alternative to Jugular Stenting in Idiopathic Intracranial Hypertension and Disturbances of Cranial Venous Outflow. J Neurol Surg B Skull Base 2017;78:158-63.

24. Dashti SR, Nakaji P, Hu YC, et al. Styloidogenic jugular venous compression syndrome: diagnosis and treatment:

Cite this article as: Bai C, Wang Z, Guan J, Jin K, Ding Y, Ji X, Meng R. Clinical characteristics and neuroimaging findings in eagle syndrome induced internal jugular vein stenosis. Ann Transl Med 2020;8(4):97. doi: 10.21037/atm.2019.12.93 case report. Neurosurgery 2012;70:E795-9.

25. Dou G, Zhang Y, Zong C, et al. Application of surgical navigation in styloidectomy for treating Eagle's syndrome. Ther Clin Risk Manag 2016;12:575-83.

26. Siniscalchi EN. Dynamic imaging in suspected Eagle syndrome. Eur Arch Otorhinolaryngol 2020;277:307.

27. Galletta K, Granata F, Longo M, et al. An unusual internal carotid artery compression as a possible cause of Eagle syndrome - A novel hypothesis and an innovative surgical technique. Surg Neurol Int 2019;10:174. 